\title{
Does Freeze-Thawing Influence the Effects of Platelet Concentrates? An In Vitro Study on Human Adipose-Derived Stem Cells
}

\author{
Caterina Ceci, MSc, ${ }^{* \dagger}$ Stefania Niada, MSc, PhD, ${ }^{* \dagger}$ Massimo Del Fabbro, MSc, PhD, ${ }^{* \dagger}$ \\ Alessandra Lolato, MSc, ${ }^{* \dagger}$ Silvio Taschieri, MD, DDS, ${ }^{* \dagger}$ Chiara Giannasi, MSc, ${ }^{* \dagger}$ and \\ Anna Teresa Brini, $M S c, P h D^{* \dagger}$
}

\begin{abstract}
Human adipose-derived stem cells (hASCs) have been proposed as a possible therapy for tissue regeneration in aesthetic, plastic, and reconstructive surgery. Today, platelet concentrates are used in a wide range of disciplines, but their storage has become a controversial aspect. The purpose of this in vitro study was to evaluate the effect of plasma rich in growth factors (PRGF), after a freeze-thawing cycle, on the proliferation and biological activity of progenitor cells involved in soft tissue healing. Different formulations of activated PRGF were added to hASCs cultured in serum-free medium. Cell proliferation was assessed by MTT test and cell count up to 7 and 12-day incubation. Osteo-differentiation ability of hASCs was also tested after 7 and 14-day incubation by alkaline phosphatase assay. The effects of 4 PRGF preparations (fresh/frozen and with/ without platelets) were compared with corresponding formulations of plasma poor in growth factors and with standard medium. hASCs cultured in the presence of platelet concentrates increased proliferation rate with respect to cells grown in standard medium without significant differences among all the tested plasma formulations on cell viability up to 12 days of culture. PRGF activity is preserved after cryopreservation and platelet-rich preparations promoted osteodifferentiation of hASCs at day 7. In conclusion, PRGF supports the proliferation and the differentiation of progenitor cells in vitro also when applied after cryopreservation. Platelet concentrates, either alone or in combination with mesenchymal stem cells, might be a valuable tool in the field of tissue regeneration.
\end{abstract}

Key Words: Adipose-derived stem cells, osteogenic differentiation, platelet concentrate, proliferation, tissue regeneration

( Craniofac Surg 2015;00: 00-00)

AQ3 From the *Dipartimento di Scienze Biomediche, Chirurgiche e OdontoiaAQ4 triche, Università degli Studi di Milano; and †IRCCS Istituto Ortopedico Galeazzi, Milano, Italy.

Received July 28, 2015.

Accepted for publication December 1, 2015.

AQ5 Address correspondence and reprint requests to Anna Teresa Brini, MSc, $\mathrm{PhD}$, Dipartimento di Scienze Biomediche, Università degli Studi di Milano, Chirurgiche e Odontoiatriche, Via Vanvitelli, 32, 20129 Milano, Italy; E-mail: anna.brini@unimi.it

The authors report no conflicts of interest.

Copyright $(\mathcal{C} 2015$ by Mutaz B. Habal, MD

ISSN: $1049-2275$

DOI: $10.1097 /$ SCS.0000000000002428 utologous fat grafting originally developed in 1983 for reconA structive purposes when Neuber applied the technique to the treatment of facial defects, but over the last few decades, it has become widely used in several areas of plastic and aesthetic surgery. ${ }^{1}$ In craniofacial surgery adipose tissue transplantation is applied to regeneration of peripheral facial nerve injures, ${ }^{2}$ to soft tissue repair after irradiation of the maxillofacial area for neoplasms, ${ }^{3}$ and to the correction of peculiar facial deformities after trauma. ${ }^{1}$ Furthermore, fat transplantation has been used in bony reconstruction for the treatment of mandibular ameloblastoma resection defects, ${ }^{4}$ the repair of large skull defects (ie, multifragment calvaria fracture),${ }^{5}$ and the mandibular distraction osteogenesis. $^{6}$ Autologous lipofilling has become popular in aesthetic and plastic surgery for many indications, including scar remodeling (especially in acne and burn patients), lip augmentation and deformity treatment, mandibular and buccal deficiency of both bone and soft tissue after tumor resection management, skin rejuvenation, wrinkle filling, lipo-facelift, malar and mental regions augmentation, ${ }^{7}$ and breast reconstruction after cancer or for deformities. ${ }^{8}$ This technique has found application also in vocal cord tissue regeneration, ${ }^{9}$ in the treatment of chronic ulceration, ${ }^{10}$ end-stage radiation dermatitis, ${ }^{11}$ alopecia, ${ }^{12}$ and other medical applications.

Stem cell therapy has shown to be promising in aesthetic and reconstructive surgery ${ }^{13}$ and several recent studies have shown adipose tissue as a convenient source of multipotent adult stem cells, called adipose-derived stem cells (ASCs). The first phase IIII clinical trials were performed by García-Olmo et al ${ }^{14}$ to investigate the efficacy and safety of expanded human adipose-derived stem cells (hASCs) in the treatment of perianal fistulae including Crohn disease. These mesenchymal stem cells possess the ability in vitro to self-maintain and to differentiate toward cells of osteogenic, ${ }^{15}$ chondrogenic,,${ }^{16}$ adipogenic, ${ }^{15}$ myogenic, ${ }^{17}$ and neurogenic lineage. ${ }^{18}$ In the last few decades the use of mesenchymal stem cells in regenerative surgical procedures is steadily growing. Mesenchymal stem cells have been harvested since long from bone marrow (BMSCs) for clinical use; however, in the last few years subcutaneous adipose tissue is being considered a convenient alternative source since it contains a large number of progenitors and it can be collected under local anesthesia with lower donor site morbidity. ${ }^{19}$ Several in vitro studies have shown the similarity between hASCs and BMSCs regarding their cellular phenotype, growth kinetics, cell senescence, and differentiation efficiency. ${ }^{20}$ On the basis of the regenerative and immunomodulatory features of hASCs, they might become useful tools for cell-mediated therapy. ${ }^{19}$ hASCs promote tissue regeneration by secreting multiple cytokines and growth factors, including insulin-like growth factor-1 (IGF-1), basic fibroblast growth factor (bFGF), hepatocyte growth factor, transforming growth factor beta 1 (TGF- $\beta 1$ ), platelet-derived growth factor (PDGF), and the proangiogenic vascular endothelial growth factor, that stimulate the recovery of damaged tissue in a paracrine 
manner. ${ }^{21}$ This mechanism is due to the recruitment of endogenous stem cells to the site and the promotion of their differentiation toward specific lineages. Due to these biological characteristics, adipose tissue, previously discarded, has recently become an invaluable stem cell source for clinical application. ${ }^{21}$ Platelet concentrates (PCs) are regenerative preparations designed for the local release of platelet growth factors into a surgical or wound site, to stimulate tissue healing or regeneration. Consequently, autogenous PCs have gained popularity as a medical treatment in a variety of applications in different fields such as dentistry, oral surgery, orthopaedics, sport medicine, dermatology, and plastic surgery, ${ }^{22}$ reducing also the patient's postoperative discomfort. Pain, swelling, and hematoma management is fundamental in any surgical procedure for preserving the patient's postsurgery quality of life. ${ }^{23}$ The rationale of using platelet concentrates is to release cytokines and growth factors (GFs), stored in $\alpha$-granules of platelets and released upon activation. GFs are involved in the proliferation of connective tissue progenitors, in the stimulation of fibroblasts and osteoblasts activity, and in promoting angiogenesis, all processes essential for tissue healing and regeneration. ${ }^{23}$

Plasma rich in growth factors (PRGF) is an autologous preparation rich in platelets, easily and rapidly obtained from a small volume of patient's blood, which, being autologous, does not promote any immune reaction nor may cause new infectious disease. ${ }^{24}$ In the PRGF the leukocytes are excluded so as to avoid any proinflammatory effects mediated by such cells. ${ }^{25}$ Therefore, patients' own plasma has been proposed as a carrier matrix for autologous growth factors where the clinical conditions require tissue repair.

Since for surgical procedures requiring multiple treatments with platelet concentrates, blood harvesting for each application is needed, PC storage has become a controversial topic. Freezethawing cycles allow an easier patient management and, in addition, frozen samples are easy to handle and consequently useful for in vitro validation of the sample functionality. On the other hand, they are supposed to reduce platelet function and GFs release and therefore may negatively affect the platelet concentrate's biological activity. ${ }^{26}$

The aim of this study was to assess the in vitro effect of PRGF after a freeze-thawing cycle, on progenitor cells involved in soft tissue regeneration, using PRGF as substitute for fetal bovine serum (FBS). In particular, vitality, cell growth, and osteo-differentiation of hASCs were evaluated. Additionally, the same experiments were repeated using plasma poor in growth factors (PPGF) to assess if the observed effects were due to plasmatic factors or to factors released by platelets.

\section{METHODS}

\section{Blood Collection and PC Preparation}

The acellular plasma of a PC was obtained using a manual protocol performed by an experienced technician. Peripheral blood from 4 young healthy volunteer donors ( 3 women and 1 man, age $23-29$ years $(26.2 \pm 2.6)$ ) was collected in $9-\mathrm{mL}$ tubes (BTI blood collecting tubes) with $3.8 \%$ sodium citrate $(\mathrm{m} / \mathrm{v})$ as anticoagulant. PC was obtained by blood centrifugation at 580 grams for 8 minutes. Blood cells stratified according to a density gradient: the bottom fraction contained erythrocytes and the top fraction contained platelets. The 2 fractions were separated by the whitish buffy coat layer, which contained most of the leukocytes. The $2 \mathrm{~mL}$ plasma fraction just above the buffy coat, containing the highest platelets concentration, was identified as plasma rich in growth factors (PRGF) and collected. The plasma fraction above the PRGF layer, presenting the lowest platelets concentration, was identified as PPGF and also collected (Fig. 1).

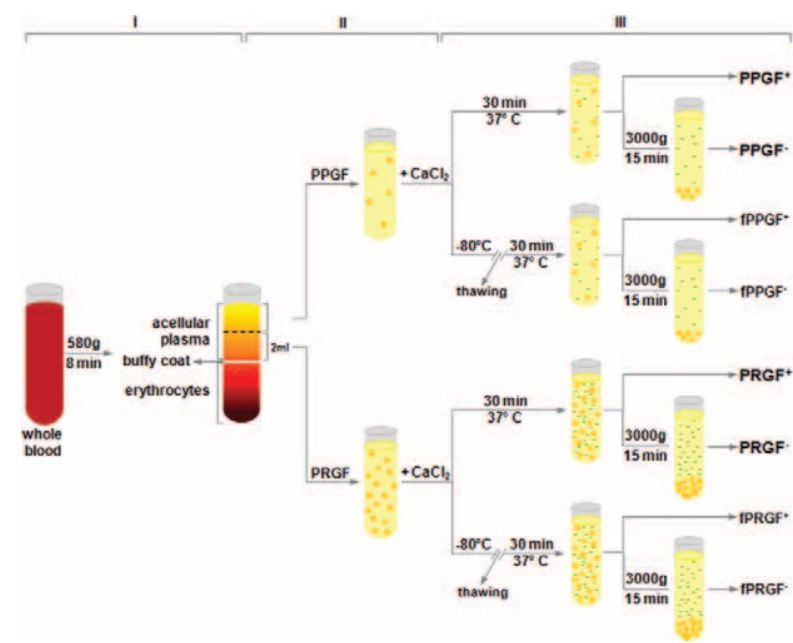

FIGURE 1. Overview of blood collection and different PC formulations preparation. Citrate-anticoagulated whole blood was centrifuged to separate blood cells into different layers, according to a density gradient. The bottom fraction contained the red blood cells, while the upper acellular plasma part contained platelets. The buffy coat layer of leukocytes separated acellular plasma and erythrocytes (part I). The platelet-rich (PRGF) and platelet-poor (PPGF) plasmas were collected and calcium chloride was added (part II). Different plasma formulations were prepared activating for 30 minutes at $37^{\circ} \mathrm{C}$ (immediately for fresh samples, after a freeze-thawing cycle for frozen ones) to initiate the release of growth factors (green dashes) by the platelets (orange spots). To remove the platelets, the clots were centrifuged and then the supernatants were collected and used for cell culture (part III).

Platelets count was obtained by a hematology analyzer (Sysmex, XE-2100, Norderstedt, Germany). The quantification of growth factors was performed by ELISA kits (R\&D Systems, Minneapolis, MI) and PDGF-AB, TGF- $\beta 1$, and vascular endothelial growth factor concentrations were determined following the manufacturer's recommendations. These GFs were chosen for analysis for their specific role in tissue healing and regeneration (Table 1). ${ }^{27}$

\section{Different Plasma Formulations Preparation}

After PRGF and PPGF fractions activation with $\mathrm{CaCl}_{2}$ solution (PRGF-Activator, BTI Biotechnology Institute, Vitoria, Spain) (Fig. 1, part II), different steps were performed to obtain 4 different PRGF preparations: fresh PRGF with platelets $\left(\mathrm{PRGF}^{+}\right)$; frozen PRGF with platelets $\left(\mathrm{fPRF}^{+}\right)$; fresh PRGF without platelets $\left(\mathrm{PRGF}^{-}\right)$, and frozen PRGF without platelets $\left(\mathrm{fPRGF}^{-}\right)$. $\mathrm{fPRGF}^{+}$ was immediately aliquoted and stored at $-80^{\circ} \mathrm{C}$ until its use. After being thawed in a water bath at $37^{\circ} \mathrm{C}$ for a few minutes, it was

TABLE 1. Description of Plasma Preparations and Concentrations of the Most Relevant Growth Factors

\begin{tabular}{lcccc}
\hline & & \multicolumn{3}{c}{ Growth Factors Concentrations } \\
\cline { 3 - 5 } Sample & $\begin{array}{c}\text { Platelet Count } \\
\text { Mean } \pm \text { SD }\left(\mathbf{1 0}^{3} / \mu \mathbf{L}\right)\end{array}$ & $\begin{array}{c}\text { PDGF-AB } \\
(\mathbf{n g} / \mathbf{m L})\end{array}$ & $\begin{array}{c}\text { TGF- } \beta \mathbf{1} \\
(\mathbf{n g} / \mathbf{m L})\end{array}$ & $\begin{array}{c}\text { VEGF } \\
(\mathbf{p g} / \mathbf{m L})\end{array}$ \\
\hline Whole blood & $248.14 \pm 31.13$ & - & - & - \\
PRGF & $434.43 \pm 108.97$ & $18.9 \pm 4.1$ & $39.2 \pm 3.5$ & $152.7 \pm 18$ \\
PPGF & $415.71 \pm 111.17$ & $1.37 \pm 0.06$ & $87.6 \pm 11.69$ & $361.1 \pm 119.5$
\end{tabular}

PDGF-AB, platelet-derived growth factor AB; PPGF, plasma poor in growth factors; PRGF, plasma rich in growth factors; SD, standard deviation; TGF- $\beta 1$, transforming growth factor beta 1; VEGF, vascular endothelial growth factor. 
incubated at $37^{\circ} \mathrm{C}$ for 30 minutes, triggering thereby platelet activation and growth factors release and then used as supplement to the culture medium. The other formulations were incubated at $37^{\circ} \mathrm{C}$ for 30 minutes and $\mathrm{PRGF}^{+}$was immediately added to the culture medium, whereas $\mathrm{PRGF}^{-}$was collected after centrifugation at $3000 \mathrm{~g}$ for 15 minutes at $4^{\circ} \mathrm{C}$ to remove platelets. Finally, aliquots of fPRGF ${ }^{-}$were stored at $-80^{\circ} \mathrm{C}$ until testing, but not longer than 24 hours, while PRGF $^{-}$was immediately used.

Similarly, 4 PPGF plasma preparations were obtained from the upper plasma fraction: fresh PPGF with platelets $\left(\mathrm{PPGF}^{+}\right)$; frozen PPGF with platelets $\left(\mathrm{fPPGF}^{+}\right)$; fresh PPGF without platelets $\left(\mathrm{PPGF}^{-}\right)$, and frozen PPGF without platelets $\left(\mathrm{fPPGF}^{-}\right)$. All PRGFs and PPGFs formulations from each donor were individually tested on human adipose-derived stem cells (Fig. 1, part III).

\section{Cell Culture}

Cells used in this study derived from plastic surgery discarded tissues of healthy donors after their written consent and Institutional Review Board approval (IRCCS Galeazzi Orthopaedic Institute PQ 7.5.125, version 3, 14.05.2012).

Human adipose-derived stem cells (hASCs) were isolated by liposuction of 15 to $40 \mathrm{~mL}(28.3 \pm 12.6 \mathrm{~mL})$ subcutaneous-adipose tissue of 3 female patients (age 22-35 years (mean $29 \pm 6.5$ years), BMI < 30). After digestion with $0.075 \%$ type I collagenase (Worthington, Lakewood, NJ) the stromal vascular fraction was separated by centrifugation (1200 grams for 10 minutes), filtered and hASCs were plated $\left(100,000\right.$ cells $\left./ \mathrm{cm}^{2}\right)$ in basal medium (DMEM) supplemented with $10 \%$ fetal bovine serum, $50 \mathrm{U} / \mathrm{mL}$ penicillin, $50 \mathrm{mg} / \mathrm{mL}$ streptomycin and $2 \mathrm{mM}$ L-glutamine. Cells were maintained in a humidified atmosphere at $37^{\circ} \mathrm{C}$ with $5 \% \mathrm{CO}_{2}$ until confluence. Plastic culture flasks-adherent hASCs were selected during subsequent passages. The fibroblast-like morphology displayed by the isolated cells was checked by phase-contrast microscopy and the doubling time was calculated as $(\mathrm{t} 2-\mathrm{t} 1) \times \ln _{2} / \ln \left(N / N_{0}\right)$, where $N$ is the number of counted cells and $N_{0}$ represents the number of plated ones. Cell viability was tested by means of 3-[4,5-dimethylthiazol-2-yl]-2,5-diphenyltetrazolium bromide (MTT) test, whereas the colony-forming unitfibroblast (CFU-F) frequency was established by counting individual colonies (of at least 20 cells) compared with the number of seeded cells. hASCs were characterized immunophenotypically by FACS Calibur flow cytometer (BD Biosciences Europe, Erembodegem, Belgium) as described previously. ${ }^{27}$ Cells were CD73, CD90, CD105, and CD271 positive and CD14 and CD45 negative. Multilineage differentiation potential into adipogenic, osteogenic, and chondrogenic differentiation was confirmed (data not shown). The hASCs purified yield after 25 days was $4.4 \times 10^{8} \pm 2.6 \times 10^{8}$.

\section{Viability and Proliferation}

MTT assay: Passage 3 to 5 cells were plated at a density of $1.5 \times 10^{4} \mathrm{cells} / \mathrm{cm}^{2}$ in 96 -well tissue-culture plates. $2.5 \%, 5 \%$ PRGF or PPGF, or $10 \%$ FBS (CTRL) were added to the cell culture medium after 24 hours of culture in serum-free medium, to allow cell cycle synchronization. After 1 and 7 days in culture, $0.5 \mathrm{mg} / \mathrm{mL}$ MTT (3-[4,5-dimethylthiazol-2-yl]-2,5-diphenyltetrazolium bromide) was added and incubated for 4 hours at $37^{\circ} \mathrm{C}$. The formazan precipitate was solubilized with $100 \%$ dimethylsulphoxide and the absorbance read at $550 \mathrm{~nm}$ with a Celbio plate reader.

Cell count: From passage 1 to 3 in culture, cells were trypsinized and plated at a density of $8 \times 10^{3}$ cells $/ \mathrm{cm}^{2}$ in 6 -well tissue-culture plates in control medium. After 24-hour starvation, culture medium was replaced by serum-free medium supplemented with either $2.5 \%$ or $5 \%$ of PRGF or PPGF formulations or $10 \%$ FBS. At days 4, 8, 12, cells were detached with $0.5 \%$ trypsin/ $0.2 \%$ EDTA and counted in a
Burker chamber considering also trypan blue exclusion. All the experiments were performed in duplicates.

\section{Osteogenic Differentiation}

From passage 1 to 3 , hASCs were plated in 24-well tissueculture plates at a density of $7.5 \times 10^{3}$ cells $/ \mathrm{cm}^{2}$ (day 7) and $5 \times 10^{3}$ cells $/ \mathrm{cm}^{2}$ (day 14) and let adhere overnight in control medium. After being washed and cultured for 24 hours in serumfree medium either $2.5 \% \mathrm{PRGF}^{+}$or $\mathrm{fPRGF}^{+}$or $10 \% \mathrm{FBS}$ (CTRL) were added. Osteogenic differentiation was induced under appropriate culture conditions (medium supplemented with $10 \mathrm{nM}$ dexamethasone, $10 \mathrm{mM}$ glycerol-2-phosphate, $150 \mu \mathrm{M}$ L-ascorbic acid2-phosphate, and $10 \mathrm{nM}$ cholecalciferol) (OSTEO).

Alkaline phosphatase (ALP) assay-enzymatic activity was measured at days 7 and 14 . Cells were lysed with $0.1 \%$ Triton $\mathrm{X}-100$ and $1 \mathrm{mM}$ p-nitrophenylphosphate in alkaline buffer (100 mM diethanolamine and $0.5 \mathrm{mM} \mathrm{MgCl} 2, \mathrm{pH} 10.5$ ) was added to the cell lysate and incubated at $37^{\circ} \mathrm{C}$. The reaction was stopped with $1 \mathrm{~N} \mathrm{NaOH}$ and absorbance read at $405 \mathrm{~nm}$ with a Wallac Victor II plate reader. ALP activity was then normalized with respect to each sample protein concentration determined by BCA protein assay (Pierce Biotechnology, Rockford, IL).

Reagents, when not otherwise indicated, were provided by Sigma-Aldrich, Milan, Italy.

\section{Statistical Analysis}

Data are expressed as means \pm SE and statistical analysis (oneway analysis of variance) was performed by using GraphPad Prism 5.03 (GraphPad Software, San Diego, CA). Differences were considered significant at $P<0.05$.

\section{RESULTS}

\section{Cell Proliferation and Viability}

A preliminary dose study for choosing the ideal PC concentration was performed. hASCs were incubated for $4,8,12$ days in medium enriched with $10 \%$ FBS (CTRL) or with $2.5 \%$ or $5 \%$ of each of the plasma formulations. The percentage of increase or decrease of cell number derived from 2 independent experiments performed with PCs from 1 donor was calculated (Table 2). The highest cell count values were obtained when hASCs were cultured in media supplemented with $5 \%$ of any PC preparations, both in the presence (Table 2A) and in the absence (Table 2B) of platelets. After 12 days cells showed an increased proliferation up to 19.8fold for PRGF and to 21-fold for PPGF with respect to the control. $2.5 \%$ PC formulations induced a lower but comparable to the control cell proliferation. Consequently, the remaining assays were performed with $5 \%$ platelet concentrate as substitute for FBS.

At day 1 , in all the $\mathrm{fPRGF}^{+}, \mathrm{PRGF}^{+}, \mathrm{fPPGF}^{+}$, and $\mathrm{PPGF}^{+}$ groups hASC viability was reduced compared with the control, suggesting that hASCs required more time to adapt to the new microenvironment. At day 7, hASC viability increased irrespectively of the supplements added, and no difference was observed anymore (Fig. 2A). A similar trend was observed by cell counting. In all GFs-enriched media, hASCs proliferated faster than in CTRL medium, confirming that these progenitors adapted easily to the human supplements. In all the tested groups, the number of hASCs collected after 12 days was about 10 times higher than the control. $\mathrm{fPRGF}^{+}$and $\mathrm{fPPGF}^{+}$groups showed higher proliferation values than the $\mathrm{PRGF}^{+}$and $\mathrm{PPGF}^{+}$, although this difference was not statistically significant. In addition, no differences in cell proliferation were observed between the $\mathrm{PRGF}^{+}$and $\mathrm{PPGF}^{+}$fractions (Fig. 2B). 


\begin{tabular}{|c|c|c|c|c|c|c|c|}
\hline \multirow[b]{2}{*}{ A } & \multirow[b]{2}{*}{ PC (\%) } & \multicolumn{2}{|c|}{4 days } & \multicolumn{2}{|c|}{8 days } & \multicolumn{2}{|c|}{12 days } \\
\hline & & 2.5 & 5 & 2.5 & 5 & 2.5 & 5 \\
\hline \multirow[t]{4}{*}{ With platelets } & PRGF & 113 & 173 & 9 & 600 & -24 & 1323 \\
\hline & fPRGF & 110 & 336 & -15 & 1138 & -78 & 2364 \\
\hline & PPGF & 28 & 278 & -43 & 465 & -70 & 1092 \\
\hline & fPPGF & 18 & 214 & 50 & 1273 & 83 & 2511 \\
\hline
\end{tabular}

\begin{tabular}{|c|c|c|c|c|c|c|c|}
\hline \multirow[b]{2}{*}{ B } & \multirow[b]{2}{*}{ PC (\%) } & \multicolumn{2}{|c|}{4 days } & \multicolumn{2}{|c|}{8 days } & \multicolumn{2}{|c|}{12 days } \\
\hline & & 2.5 & 5 & 2.5 & 5 & 2.5 & 5 \\
\hline \multirow[t]{4}{*}{ Without platelets } & PRGF & 32 & 37 & -12 & 51 & -27 & 84 \\
\hline & fPRGF & 65 & 167 & 29 & 389 & 77 & 1915 \\
\hline & PPGF & -37 & -29 & -48 & -56 & -74 & -41 \\
\hline & fPPGF & -25 & 11 & -56 & 52 & -45 & 119 \\
\hline
\end{tabular}

fPPGF, frozen plasma poor in growth factors; fPRGF, frozen plasma rich in growth factors; PC, platelet concentrate; PPGF, plasma poor in growth factors; PRGF, plasma rich in growth factors.

$\mathrm{fPRGF}^{-}, \mathrm{PRGF}^{-}, \mathrm{fPPGF}^{-}$, and $\mathrm{PPGF}^{-}$did not affect hASC viability despite a large variability among samples (Fig. 2C). On the other hand, hASC proliferation increased only with $\mathrm{PRGF}^{-}$, $\mathrm{PRGF}^{-}$, and $\mathrm{fPPGF}^{-}$, whereas $\mathrm{PPGF}^{-}$displayed similar results as $10 \%$ FBS (Fig. 2D). At day 4, the presence of platelets seemed to have a higher cell proliferation-inducing effect, in particular after treatment with 5\% PCs, than that observed in their absence (Fig. 2B and D).

This result was also confirmed by phase contrast microscopy that revealed a higher number of hASCs and the typical elongated fibroblastic appearance when cells were grown in the presence of any plasma formulations with respect to the control condition (Fig. 2E).

The effect of fPRGF + from several donors and on different hASC populations was also analyzed. The interdonor variability among platelet concentrates and hASC populations was less pronounced at early time points compared with later ones, and the marked effect of fPRGF+, independently of its donor and of the responder cells (different hASCs populations), was confirmed at a later time point (Fig. 3).

\section{Osteogenic Differentiation}

ALP activity was determined in $2 \mathrm{hASC}$ populations (ASC-I and ASC-II) osteo-differentiated for 7 and 14 days in the presence of either $2.5 \% \mathrm{PRGF}^{+}$or $\mathrm{fPRGF}^{+}$. After 7 days ALP activity considerably increased when platelet concentrates were present (Fig. 4A). Cells cultured with both $\mathrm{PRGF}^{+}$and $\mathrm{fPRGF}^{+}$exhibited higher levels of this marker compared with those cultured with FBS. In addition, in the absence of osteo-differentiative stimuli ALP activity was also induced. At day 14 , the effect was reduced with respect to day 7 , but still present (Fig. 4B). In parallel, calcium deposition tested by alizarin red staining (ARS) was also increased after 14 days of differentiation by the presence of both $\mathrm{PRGF}^{+}$and fPRGF $^{+}$(data not shown).

\section{DISCUSSION}

The use of autologous platelet concentrate has spread in different medical conditions for regenerative purpose. ${ }^{25}$ Its effects on tissue
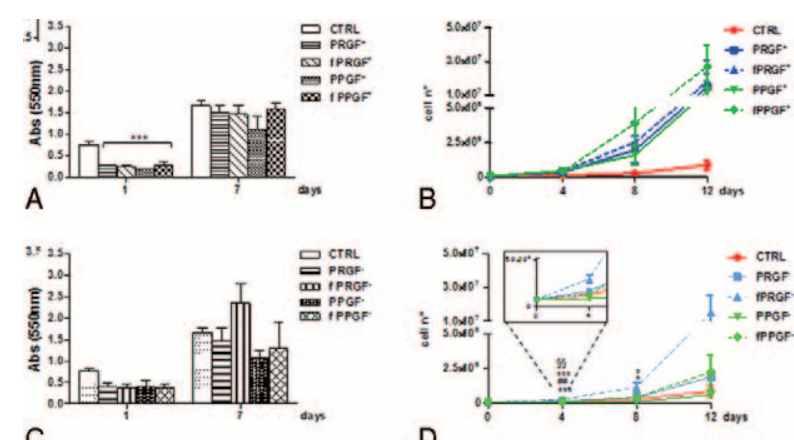

C
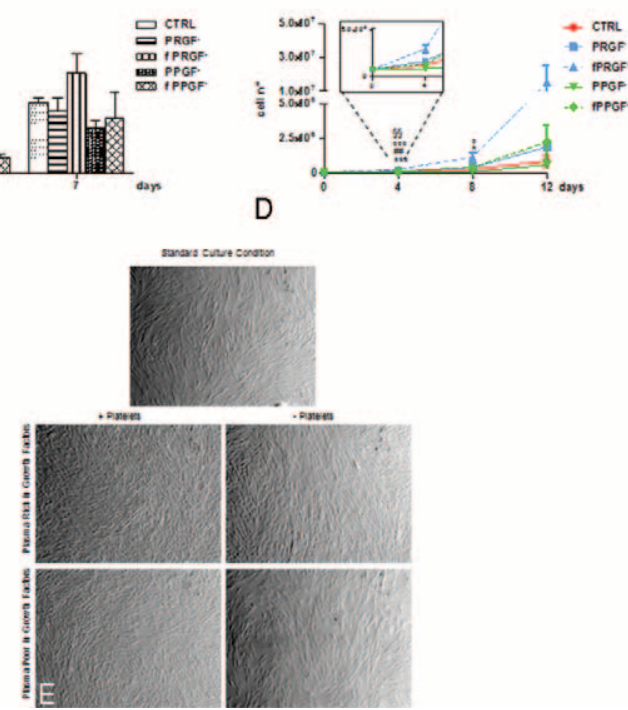

FIGURE 2. Effect of plasma rich in growth factors (PRGF) and plasma poor in growth factors (PPGF) on human adipose-derived stem cell (hASC) viability and proliferation. Evaluation of cell viability with $5 \%$ fresh and frozen (f) PRGF, PPGF in the presence $(+)(A)$ and in the absence $(-)(C)$ of platelets. Data are shown as means \pm SE of 3 independent experiments performed with platelets

concentrates derived from blood of 3 donors. $* * * P<0.001$ treated versus control value. hASC proliferation with $5 \%$ fresh and frozen (f) PRGF, PPGF with (B) and without (D) platelets. Data are shown as the means \pm SE (standard error) of 3 independent experiments. At day 4 in the absence of platelets $* * * P<0.001 \mathrm{fPRGF}^{-}$versus CTRL (control); \#\#P $<0.01 \mathrm{fPRGF}^{-}$versus $\mathrm{PRGF}^{-}$. ${ }^{000} P<0.001 \mathrm{fPRGF}^{-}$versus $\mathrm{PPGF}^{-} ; \S \S P<0.01 \mathrm{fPRGF}^{-}$versus $\mathrm{fPPGF}^{-}$. At day 8 in the absence of platelets $* P<0.05 \mathrm{fPRGF}^{-}$versus $\mathrm{CTRL}$ (control); ${ }^{\circ} P<0.05$ fPRGF $^{-}$versus PPGF ${ }^{-}$(D). Higher magnification of statistically significant difference in cell proliferation at day 4 (inset). Representative phase contrast photomicrographs illustrating the proliferation rate of hASCs grown on a tissue culture plate for 4 days in standard condition (CTRL) and in media supplemented with $\mathrm{PRGF}^{+}, \mathrm{PRGF}^{-}, \mathrm{PPGF}^{+}, \mathrm{PPGF}^{-}(\times 40$ magnification $)(\mathrm{E})$

regeneration have been shown in dentistry, oral implantology, orthopaedics, sports medicine, and treatment of skin disorders. ${ }^{25}$ Currently, different techniques to obtain platelet concentrates are available, but each method may lead to preparations that may differ in platelet concentration, leukocyte, and fibrin content. ${ }^{28}$ PRGF is a modified pure platelet-rich plasma, introduced by Anitua in $1999 .{ }^{24}$ The main difference with platelet-rich plasma is that according to the Anitua's protocol, leukocytes are intentionally excluded to avoid proinflammatory effects of proteases and acid hydrolases contained in white blood cells. ${ }^{25}$ In this study, we demonstrated that PRGF preserves the ability of inducing in vitro proliferation and osteogenic differentiation of hASCs, even after freezethawing cycle.

The effect of PRGF was compared to control culture condition to test if PRGF could be a possible alternative to FBS supplement in cell culture media.

To date, little is known about the in vitro effect of platelet concentrates on cell function. Despite the low number of studies that investigated the effect of platelet concentrates on hASCs, the effectiveness of PCs in combination with hASCs on cell stimulation and tissue healing has been reported. ${ }^{3}$ This was also confirmed in our study in which FBS was completely substituted by PC. In this 

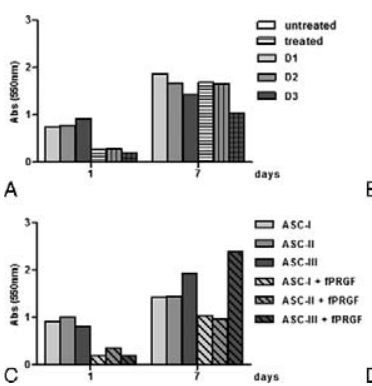

B
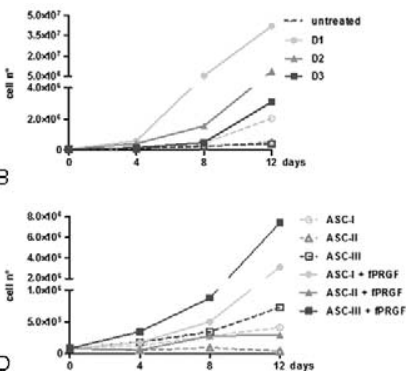

FIGURE 3. (A, B) Effect of $5 \%$ frozen plasma rich in growth factors with platelets $\left(\mathrm{fPRGF}^{+}\right)$from different donors and (C, D) on different human adipose-derived stem cells (hASC) populations. (A) Cell viability (MTT assay) and (B) proliferation (cell count) of a single hASC population (I) cultured with $3 \mathrm{fPRGF}^{+}$samples (D1, D2, D3). (C) Viability and (D) proliferation of 3 hASC populations (I, II, III) cultured with a $\mathrm{fPRFF}^{+}$sample. A representative experiment for each condition is shown.

study, mesenchymal stem cells and platelet concentrate were not harvested from the same donors. This choice was supported by a previous study that reported that hASCs grown in the presence of both human autologous and heterologous sera increased their proliferation rate compared with standard culture condition, ${ }^{29}$ and especially with the absence of leukocytes from PRGF with a consequent reduced risk of immune reaction between the PC cells and the cultured ones. Although culture media have been supplemented with a lower concentration of plasma formulations compared with other studies, ${ }^{30}$ after a short adaptation period, cell viability and proliferation were comparable to the control ones in all the tested groups. Interestingly, we observed similar effects mediated by PRGF and PPGF (both fresh and frozen). One possible explanation of this result could be the non-direct-correlation between platelet count and growth factors concentration and the unclear relationship between platelet count and cellular response, as reported by Zimmermann et $\mathrm{al}^{31}$ and Eppley et $\mathrm{al}^{22}$ On the other hand, other authors reported that platelet count positively correlates with the quantity of growth factors released. ${ }^{32}$ Due to the high number of GFs contained in $\alpha$-granules, it is very difficult, though it could be relevant, to measure the levels of each of them. In principle, several factors should be considered that may affect the establishment of precise correlations. For example, the content of GFs stored in, and the amount of GFs released by each cell, may
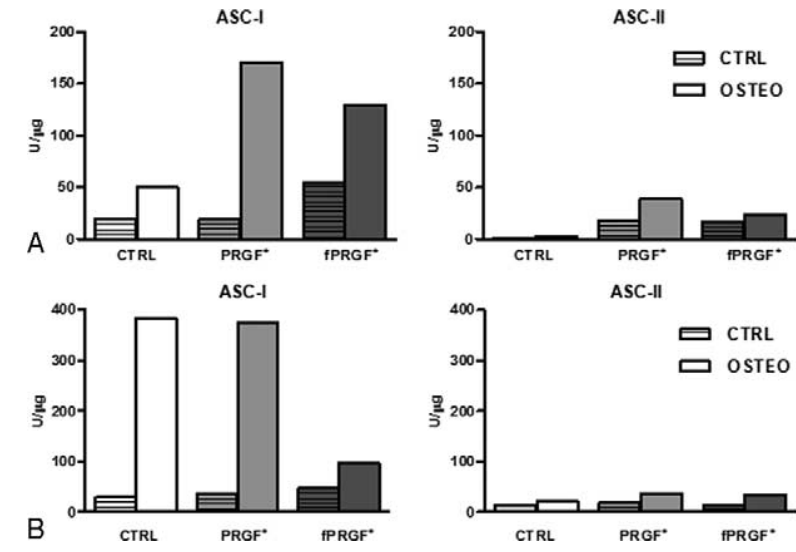

FIGURE 4. ALP activity of 2 human adipose-derived stem cell (hASC) populations (I, II) osteo-induced in the presence of $2.5 \%$ of frozen plasma rich in growth factors with platelets $\left(\mathrm{fPRGF}^{+}\right)$and fresh plasma rich in growth factors with platelets $\left(\mathrm{PRGF}^{+}\right)$for $7(\mathrm{~A})$ and 14 (B) days. ALP activity is expressed as $\mathrm{U} / \mathrm{mg}$ proteins. A single experiment for each condition is shown. ALP, alkaline phosphatise. vary. We should keep in mind that platelets derive from fragmentation of larger cells, the megakaryocites, and this process does not always generate identical cells. Furthermore, platelets activation, which causes discharge of platelets granules content by exocytosis in the extracellular space, may not occur in the same way and simultaneously for all the platelets involved, especially in the in vivo situation. The GFs' release kinetics may vary according to the interaction of platelets with the surrounding extracellular matrix and/or with other cells and biological mediators involved in early healing events. ${ }^{33}$

The possibility of storing platelet concentrates and the effects of freeze-thawing cycles on platelet activity are poorly understood and still under debate. Some researchers consider freeze-thawing a procedure that could impair platelet function and lifespan, alter the GFs' release pattern, promote the pyrogenic cytokines accumulation, and increase the risk of bacterial proliferation, ${ }^{26}$ whereas other authors believe that it does not affect the platelet concentrate's final properties. ${ }^{34}$

In this study, we demonstrated that cryopreservation can be regarded as a safe procedure to store platelet concentrates because both fresh and frozen samples showed similar biological activities. Despite Zimmermann et $\mathrm{al}^{35}$ reporting that freezing and thawing damaged platelet membranes, causing extensive degranulation and increase of GFs content in plasma, Perut et $\mathrm{a}^{36}$ showed that this procedure reduced platelet number but did not affect the GFs release. This result was also confirmed by Roffi et $\mathrm{al}^{37}$ whose study underlined that although freezing and thawing affected the GFs' release kinetics, fresh and frozen PCs did not significantly differ in their ability to induce cell proliferation. This storage option could simplify the management of patients undergoing multiple PCs application sessions, keeping however in mind that biological results cannot always be directly translated into clinical outcomes. ${ }^{37}$ Although the aim of this study was just to compare the effect of fresh and frozen platelet concentrates, as freezing could be considered a sample storage option, another interesting issue is the possibility that a prolonged storage time could have a strong influence on the results of platelet concentrates interactions with hASCs. Currently, there is a lack of data available about the preservation of biological activity potency of PCs after different storage times, but Anitua et $\mathrm{al}^{38}$ demonstrated that PRGF eye drops stored at $-20^{\circ} \mathrm{C}$ for 15,30 , and 90 days maintained the same concentrations of the main proteins and growth factors and did not affect the in vitro effects of the eye drops; therefore, this point will be of great interest for future investigations.

In the present study, PCs were activated with calcium chloride before freezing to induce releasing of the growth factors. Hence, freezing was used only as a GFs storage method and not to produce the so-called "platelet lysates"; in fact, the latter derived by mechanical disruption of platelet concentrates via freeze-thawing cycles with no chemical activation. ${ }^{39}$ To assure the clinician on the effectiveness of a regenerative treatment, it would be useful to analyze the bioactivity of the patient's own platelet concentrate, as the GFs levels might vary among donors, with variable effects on tissue healing. We believe that combining in vitro tests with the clinical application might either encourage or save from using autologous platelet concentrates in selected patients. Despite platelet count and PC growth factors content being likely influenced by the donor's age, sex, and thrombocyte count, Weibrich et $\mathrm{al}^{40}$ did not find substantial variation in individual growth factors content and the donor's biological condition. In fact, our results indicated an improvement of the biological analyzed parameters despite a mild interdonor variability of the tested PCs.

To try to elucidate the PRGF potential in bone regeneration, its pro-osteogenic activity was evaluated by ALP activity, an early marker of osteogenic differentiation. Cells were maintained in 
media supplemented with $2.5 \%$ platelet concentrates, indicating that even such low amount of PC was adequate to induce cell proliferation. ALP activity assay showed that 2.5\% PRGF, both fresh and frozen, is capable of supporting in vitro differentiation of hASCs suggesting that platelet concentrates contain per se osteoinductive factors that might activate some early steps of the osteodifferentiative process of mesenchymal cells. Our results are consistent with previous findings that demonstrated that the adjunct of platelet concentrates to culture medium promotes the differentiation of various cell types. ${ }^{36,41}$

In this study, we did not analyze platelet concentrates from elderly donors, which hypothetically could show some variability with respect to the plasma formulations from young volunteers. This point should be further investigated in subsequent studies. All types of PRGFs enhanced the cell proliferative and differentiative capacity in vitro suggesting also that the concentrated cocktail of bioactive components present in platelet concentrates could be a valid substitute of FBS as supplement for culture medium as also demonstrated by Creeper et al. ${ }^{41}$ Further studies will focus on the proteomic analysis of the plasma concentrates with the idea to deplete some factors involved in the healing process, to determine their specific biological role.

In some instances, the results obtained here might suggest that the presence of platelets is not always quite important, which is misleading. We must take into account that the in vitro situation is only partially comparable to the in vivo condition. In fact, the biological environment of a healing tissue is extremely more complex and undergoes continuous modifications regarding cell composition, tissue $\mathrm{pH}$, oxygen content. Moreover, in vivo, a far greater number of signaling molecules are involved and may interact with platelets and other cells in orchestrating the healing process. Recent proteomics studies showed that more than 4000 soluble factors are contained into and released by the platelet granules and for most of them the actual role is still unknown. ${ }^{42}$ Additional studies are needed to shed light on the actual role of platelets in the tissue regeneration process.

In conclusion, this study demonstrated that cryopreservation does not affect PC stimulating effect on cell proliferation and differentiation and that hASCs might be an ideal tool for assessing the activity of platelet concentrates. Consequently, we provide evidence to support platelet concentrates in association with hASCs as an attractive adjunct for reconstructive and aesthetic surgery.

\section{ACKNOWLEDGMENTS}

This study was funded by Department of Biomedical, Surgical and Dental Sciences, Università degli Studi di Milano, and by IRCCS Istituto Ortopedico Galeazzi (RC no. L1007). The authors thank Dr E. Arrigoni for the technical support.

\section{REFERENCES}

1. Arcuri F, Brucoli M, Baragiotta N, et al. The role of fat grafting in the treatment of posttraumatic maxillofacial deformities. Craniomaxillofac Trauma Reconstr 2013;6:121-126

2. Euler de Souza Lucena E, Guzen FP, Lopes de Paiva Cavalcanti JR, et al. Experimental considerations concerning the use of stem cells and tissue engineering for facial nerve regeneration: a systematic review. J Oral Maxillofac Surg 2014;72:1001-1012

3. Chen Y, Niu Z, Xue Y, et al. Improvement in the repair of defects in maxillofacial soft tissue in irradiated minipigs by a mixture of adiposederived stem cells and platelet-rich fibrin. Br J Oral Maxillofac Surg 2014;52:740-745

4. Sándor GK, Tuovinen VJ, Wolff J, et al. Adipose stem cell tissueengineered construct used to treat large anterior mandibular defect: a case report and review of the clinical application of good manufacturing practice-level adipose stem cells for bone regeneration. J Oral Maxillofac Surg 2013;71:938-950
5. Lendeckel S, Jödicke A, Christophis P, et al. Autologous stem cells (adipose) and fibrin glue used to treat widespread traumatic calvarial defects: case report. J Craniomaxillofac Surg 2004;32: 370-373

6. Hwang YJ, Choi JY. Addition of mesenchymal stem cells to the scaffold of platelet-rich plasma is beneficial for the reduction of the consolidation period in mandibular distraction osteogenesis. J Oral Maxillofac Surg 2010;68:1112-1124

7. Coleman SR. Structural fat grafting: more than a permanent filler. Plast Reconstr Surg 2006;118:S108-S120

8. Coleman SR, Saboeiro AP. Fat grafting to the breast revisited: safety and efficacy. Plast Reconstr Surg 2007;119:775-785

9. Mazzola RF, Cantarella G, Torretta S, et al. Autologous fat injection to face and neck: from soft tissue augmentation to regenerative medicine. Acta Otorhinolaryngol Ital 2011;31:59-69

10. Marangi GF, Pallara T, Cagli B, et al. Treatment of early-stage pressure AQ6 ulcers by using autologous adipose tissue grafts. Plast Surg Int 2014817283

11. Rigotti G, Marchi A, Galiè M, et al. Clinical treatment of radiotherapy tissue damage by lipoaspirate transplant: a healing process mediated by adipose-derived adult stem cells. Plast Reconstr Surg 2007;119: 1409-1422

12. Dini M, Mori A, Quattrini Li A. Eyebrow regrowth in patient with atrophic scarring alopecia treated with an autologous fat graft. Dermatol Surg 2014;40:926-928

13. Wu Y, Chen L, Scott PG, et al. Mesenchymal stem cells enhance wound healing through differentiation and angiogenesis. Stem Cells 2007;25:2648-2659

14. García-Olmo D, Herreros D, De-La-Quintana P, et al. Adiposederived stem cells in Crohn's rectovaginal fistula. Case Rep Med 2010961758

15. De Girolamo L, Stanco D, Salvatori L, et al. Stemness and osteogenic and adipogenic potential are differently impaired in subcutaneous and visceral adipose derived stem cells (ASCs) isolated from obese donors. Int J Immunopathol Pharmacol 2013;26:S11-S21

16. Rada T, Carvalho PP, Santos TC, et al. Chondrogenic potential of two hASCs subpopulations loaded onto gellan gum hydrogel evaluated in a nude mice model. Curr Stem Cell Res Ther 2013;8:357-364

17. Nishida M, Li TS, Hirata K, et al. Improvement of cardiac function by bone marrow cell implantation in a rat hypoperfusion heart model. Ann Thorac Surg 2003;75:768-773

18. Safford KM, Hicok KC, Safford SD, et al. Neurogenic differentiation of murine and human adipose-derived stromal cells. Biochem Biophys Res Commun 2002;294:371-379

19. Kakudo N, Shimotsuma A, Kusumoto K. Fibroblast growth factor-2 stimulates adipogenic differentiation of human adipose-derived stem cells. Biochem Biophys Res Commun 2007;359:239-244

20. Zuk PA, Zhu M, Ashjian P, et al. Human adipose tissue is a source of multipotent stem cells. Mol Biol Cell 2002;13:4279-4295

21. Philips BJ, Marra KG, Rubin JP. Healing of grafted adipose tissue. Current clinical applications of adipose-derived stem cells for breast and face reconstruction. Wound Repair Regen 2014;22:S11-S13

22. Eppley BL, Woodell JE, Higgins J. Platelet quantification and growth factor analysis from platelet-rich plasma: implications for wound healing. Plast Reconstr Surg 2004;114:1502-1508

23. Del Fabbro M, Bortolin M, Taschieri S, et al. Effect of autologous growth factors in maxillary sinus augmentation: a systematic review. Clin Implant Dent Relat Res 2013;15:205-216

24. Anitua E. Plasma rich in growth factors: preliminary results of use in the preparation of future sites for implants. Int J Oral Maxillofac Implants 1999; 14:529-535

25. Anitua E, Sánchez M, Orive G, et al. Delivering growth factors for therapeutics. Trends Pharmacol Sci 2008;29:37-41

26. Kon E, Filardo G, Di Matteo B, et al. PRP for the treatment of cartilage pathology. Open Orthop J 2013;7:120-128

27. Alsousou J, Thompson M, Hulley P, et al. The biology of platelet-rich plasma and its application in trauma and orthopaedic surgery: a review of the literature. J Bone Joint Surg Br 2009;91:987-996

28. Dohan Ehrenfest DM, Rasmusson L, Albrektsson T. Classification of platelet concentrates: from pure platelet-rich plasma (P-PRP) to leucocyte- and platelet-rich fibrin (L-PRF). Trends Biotechnol 2009;27:158-167 
29. Broccaioli E, Niada S, Rasperini G, et al. Mesenchymal stem cells from Bichat's fat pad: in vitro comparison with adipose-derived stem cells from subcutaneous tissue. Biores Open Access 2013;2:107-117

30. Anitua E, Tejero R, Zalduendo MM, et al. Plasma rich in growth factors promotes bone tissue regeneration by stimulating proliferation, migration, and autocrine secretion in primary human osteoblasts. $J$ Periodontol 2013;84:1180-1190

31. Zimmermann R, Jakubietz R, Jakubietz M, et al. Different preparation methods to obtain platelet components as a source of growth factors for local application. Transfusion 2001;41:1217-1224

32. Sundman EA, Cole BJ, Fortier LA. Growth factor and catabolic cytokine concentrations are influenced by the cellular composition of platelet-rich plasma. Am J Sports Med 2011;39:2135-2140

33. Anitua E, Andia I, Ardanza B, et al. Autologous platelets as a source of proteins for healing and tissue regeneration. Thromb Haemost 2004;91:4-15

34. Dhillon M, Patel S, Bali K. Platelet-rich plasma intra-articular knee injections for the treatment of degenerative cartilage lesions and osteoarthritis. Knee Surg Sports Traumatol Arthrosc 2011;19:863-864

35. Zimmermann R, Arnold D, Strasser E, et al. Sample preparation technique and white cell content influence the detectable levels of growth factors in platelet concentrates. Vox Sang 2003;85:283-289
36. Perut F, Filardo G, Mariani E, et al. Preparation method and growth factor content of platelet concentrate influence the osteogenic differentiation of bone marrow stromal cells. Cytotherapy 2013;15:830-839

37. Roffi A, Filardo G, Assirelli E, et al. Does platelet-rich plasma freezethawing influence growth factor release and their effects on chondrocytes and synoviocytes? Biomed Res Int 2014692913

38. Anitua E, Muruzabal F, Pino A, et al. Biological stability of plasma rich in growth factors eye drops after storage of 3 months. Cornea 2013;32:1380-1386

39. Bieback K. Platelet lysate as replacement for fetal bovine serum in mesenchymal stromal cell cultures. Transfus Med Hemother 2013;40:326-335

40. Weibrich G, Kleis WK, Hafner G, et al. Growth factor levels in plateletrich plasma and correlations with donor age, sex, and platelet count. $J$ Craniomaxillofac Surg 2002;30:97-102

41. Creeper F, Lichanska AM, Marshall RI, et al. The effect of platelet-rich plasma on osteoblast and periodontal ligament cell migration, proliferation and differentiation. J Periodontal Res 2009;44:258-265

42. Burkhart JM, Vaudel M, Gambaryan S, et al. The first comprehensive and quantitative analysis of human platelet protein composition allows the comparative analysis of structural and functional pathways. Blood 2012;120:e73-e82 


\section{SCS}

Journal of Craniofacial Surgery

Manuscript No. 15-1130

Dear Author,

During the preparation of your manuscript for typesetting, some queries have arisen. These are listed below. Please check your typeset proof carefully and mark any corrections in the margin as neatly as possible or compile them as a separate list. This form should then be returned with your marked proof/list of corrections to the Production Editor.

\section{QUERIES: to be answered by AUTHOR}

\begin{tabular}{|l|l|l|}
\hline QUERY NO. & \multicolumn{1}{c|}{ QUERY DETAILS } & \multicolumn{1}{c|}{ RESPONSE } \\
\hline$<$ AQ1 $>$ & $\begin{array}{l}\text { Please confirm whether surnames/family } \\
\text { names (red) have been identified } \\
\text { correctly in the author byline. } \\
\text { Please check the running head for } \\
\text { correctness. }\end{array}$ \\
$<$ AQ3 $>\quad \begin{array}{l}\text { Please verify the accuracy of both the } \\
\text { author affiliations section and the } \\
\text { conflicts of interest section. }\end{array}$ \\
$<$ Alease provide the departments and \\
$<$ institutions names in English. \\
$<$ Alease check the correspondence \\
address for its correctness. \\
Please provide volume in refs. (10, 14, \\
37).
\end{tabular}

